\title{
Measuring Quality of Life in Pediatric Patients With Inflammatory Bowel Disease: Psychometric and Clinical Characteristics
}

\section{Citation}

Perrin, James M, Karen Kuhlthau, Aziz Chughtai, Diane Romm, Barbara S Kirschner, George D Ferry, Stanley A Cohen, et al. 2008. "Measuring Quality of Life in Pediatric Patients With Inflammatory Bowel Disease: Psychometric and Clinical Characteristics." Journal of Pediatric Gastroenterology and Nutrition 46 (2) (February): 164-171. doi:10.1097/mpg.0b013e31812f7f4e.

\section{Published Version}

doi:10.1097/MPG.0b013e31812f7f4e

\section{Permanent link}

http://nrs.harvard.edu/urn-3:HUL.InstRepos:30207938

\section{Terms of Use}

This article was downloaded from Harvard University's DASH repository, and is made available under the terms and conditions applicable to Other Posted Material, as set forth at http:// nrs.harvard.edu/urn-3:HUL.InstRepos:dash.current.terms-of-use\#LAA

\section{Share Your Story}

The Harvard community has made this article openly available.

Please share how this access benefits you. Submit a story.

Accessibility 


\title{
Measuring Quality of Life in Pediatric Patients With Inflammatory Bowel Disease: Psychometric and Clinical Characteristics
}

\author{
James M. Perrin ${ }^{*}$, Karen Kuhlthau ${ }^{*}$, Aziz Chughtai ${ }^{*}$, Diane Romm ${ }^{\star}$, Barbara S. Kirschner†, \\ George D. Ferry $\ddagger$, Stanley A. Cohen $\S$, Benjamin D. Gold $\S$, Melvin B. Heyman \\ Baldassanoll, and Harland S. Winter \\ *Department of Pediatrics, MassGeneral Hospital for Children, Boston \\ †University of Chicago Comer Children's Hospital, Chicago, Illinois \\ ‡Baylor College of Medicine, Houston, Texas \\ $\S$ Children's Healthcare of Atlanta, Atlanta, Georgia \\ IUniversity of California, San Francisco \\ "The Children's Hospital of Philadelphia, Philadelphia, Pennsylvania
}

\begin{abstract}
Objective-To extend development of a pediatric inflammatory bowel disease (IBD) healthrelated quality of life (HRQoL) measure by determining its factor structure and associations of factors with generic HRQoL measures and clinical variables.

Patients and Methods-Cross-sectional survey of children and adolescents ages 8 years to 18 years and their parents attending any of 6 US IBD centers, recruited from either existing registry of age-eligible subjects or visits to participating centers. The survey included generic (Pediatric Quality of Life Inventory) and IBD-specific (Impact Questionnaire) quality of life measures, disease activity, and other clinical indicators. We carried out factor analysis of Impact responses, comparing resulting factors with results on the generic HRQoL and the clinical measures.
\end{abstract}

Results-We included 220 subjects (161 with Crohn disease and 59 with ulcerative colitis). Initial confirmatory factor analysis did not support the 6 proposed Impact domains. Exploratory factor analysis indicated 4 factors with good to excellent reliability for IBD responses: general well-being and symptoms, emotional functioning, social interactions, and body image. Two items did not load well on any factor. The 4 factors correlated well with the Pediatric Quality of Life Inventory and subscales. Children with higher disease activity scores and other indicators of clinical activity reported lower HRQoL.

Conclusions-This study provides further characteristics of a HRQoL measure specific to pediatric IBD and indicates ways to score the measure based on the resulting factor structure. The measure correlates appropriately with generic HRQoL measures and clinical severity indicators.

\section{Keywords}

Crohn disease; Ulcerative colitis; Well-being; Disease activity; Functional status

(C) 2008 by European Society for Pediatric Gastroenterology, Hepatology, and Nutrition and North American Society for Pediatric Gastroenterology, Hepatology, and Nutrition

Address correspondence and reprint requests to James Perrin, MD, 50 Staniford Street, Suite 901, Boston, MA 02114 (jperrin@ partners.org).

The authors report no conflicts of interest. 
With improvements in chronic illness care and the shift of therapeutic research from improving survival to improving long-term outcomes, investigators have recognized the importance of developing broader measures of child and adolescent health status, and providers have wanted to understand the factors that have an impact on adaptation to chronic illness (1). Health-related (HR) quality of life (QoL) describes assessments that complement usual clinical measures, generally assessing patients' views of their own health. Quality of life generally reflects a personal assessment of broad areas of life status and experiences. HRQoL measures include both generic health-related measures (eg, physical stamina affected by chronic illness) and condition-specific measures (eg, gastrointestinal symptoms among adolescents with inflammatory bowel disease [IBD]). HRQoL measures have a role in the monitoring of changes in conditions and the effects of new treatments.

More work has examined QoL among adults with IBD than among children and adolescents, although $20 \%$ to $25 \%$ of IBD manifests during childhood $(2,3)$. Adults with IBD rate their overall well-being as good and indicate that the condition has not significantly disrupted their work or personal lives $(4,5)$. Adults with Crohn disease (CD) generally report worse HRQoL than those with ulcerative colitis (UC), with HRQoL also worse with active disease than with disease in remission (6). Previous pediatric studies have focused mainly on psychosocial factors as they relate to illness among children and adolescents with IBD, and have yielded inconsistent findings depending on the comparison group (7-9). Studies comparing children and adolescents with IBD with control populations have, as with other chronic conditions, indicated higher rates of psychological disturbance, including especially anxiety and depression, and problems in social functioning and self-esteem (10-12). Ferry's analytical framework for the measurement of HRQoL in children and adolescents with IBD focused on similar domains as those for generic measures: physical/occupational functioning, psychological state, social interaction, and physical (somatic) symptoms (13). Collaboration among investigators in Canada and the Netherlands and elsewhere has led to the development of a condition-specific measure of HRQoL for children and adolescents with IBD, the Impact Questionnaire. Its topics developed initially from interviews with children and adolescents ages 8 to 17 years with IBD (14), followed by reduction in items through ratings of importance by respondents with IBD, leading initially to a 33-item measure, to which 2 items were added to enhance postulated domains. These investigators developed a consensus postulating 6 domains: bowel symptoms, systemic symptoms, social/ functional concerns, body image, test and treatment concerns, and emotional concerns. No analytical process, such as factor analysis, was used in constructing the domains or assigning items. The measure indicates differences by condition, with those with $\mathrm{CD}$ having greater concerns about body image, fatigue, and interference with usual daily activities and those with UC raising more concerns about bowel symptoms and noting a broader array of emotional responses (14). This instrument has demonstrated good face validity (15), but has performed little further examination of the domains or comparison with other measures of HRQoL, other than a study of 42 Dutch children with IBD showing correlations between 0.45 and 0.84 for the domain scales with a generic HRQoL measure (16) and another study correlating the emotional domain with related domains on the Piers-Harris and the Child Health Questionnaire (17).

The goals of this study, involving a consortium of pediatric IBD centers, were to extend evaluation of the Impact Questionnaire in a larger population and through factor analysis. We expected that confirmatory factor analysis would support the original 6 domains proposed by the developers of the measure. In addition, we compared the IBD-specific QoL findings with those on a more generic HRQoL measure and with measures of severity of IBD, as further validation of the measure. We hypothesized that the IBD measure would correlate well with broader measures of HRQoL. Furthermore, we expected that children 
and adolescents with more severe or active disease would report lower QoL. Based on previous research, we also expected that subjects with CD would report worse HRQoL.

\section{PATIENTS AND METHODS}

This study sampled children diagnosed with UC or CD followed at 1 of 6 pediatric gastroenterology centers: Massachusetts General Hospital for Children; Emory University and Children's Healthcare of Atlanta; University of Chicago Comer Children's Hospital; University of California, San Francisco; Children's Hospital of Philadelphia; and Baylor College of Medicine. Children were eligible for the study if they were between the ages of 8 and 17 years (up to 18th birthday); diagnosed with UC or CD more than 6 months before the study; had no other non-IBD related chronic conditions; and had no major limitation in cognitive ability that would affect their ability to respond to the survey instruments. We used 2 methods of sampling. First, these centers had enrolled approximately 1800 children with IBD in a previously described consortium database (18). Parents of these children had agreed to participate in research across the sites when entered into the database. We used a table of random numbers to select a sample of those patients with active records in the database who met the study entry criteria, with the intent of having each site contribute subjects according to the size of the practice at that site. Iffamilies had more than 1 child with IBD, then we selected 1 child at random. Second, we prospectively identified additional patients with IBD who were scheduled for clinical visits in 2 sites where the database-identified subjects yielded too few subjects from the consortium database, and site clinicians approached these subjects for participation on these visits.

Parents of eligible children either received a letter, along with survey materials, mailed from their attending gastroenterologist, describing the study and requesting participation; or received the same letter during a routine clinical visit (as a handout) from the center director and clinical coordinator. Staff contacted nonrespondents of mailed materials by telephone after 3 weeks. Informed consent was obtained from each parent and child and adolescent participants gave informed assent. This study had institutional review board approval from each collaborating site.

\section{MEASURES}

\section{IBD-specific Quality of Life}

We used the Impact-II Questionnaire developed by Griffiths, Otley, et al $(14,17)$ to examine aspects of QoL in IBD-specific areas in children 8 years and older. As noted above, this measure consists of 35 items, grouped into 6 domains. The Impact Questionnaire has high internal reliability for the measure in general (0.96) (17) and for the 6 domains (Cronbach $\alpha$ $>0.70$ for all but body image and treatment-related concerns) (19). The Impact-II uses a visual analog scale for all answers, in which each question is oriented with higher scores to the right. Items are scored by the number of centimeters over the total length of the line; higher scores indicate better HRQoL.

\section{Generic Quality of Life}

We used the Pediatric Quality of Life Inventory (PedsQL) developed by Varni et al (20) as the measure of generic QoL. This measure captures both parent and child perspectives on child HRQoL, takes less than 5 minutes to complete, comes in English and Spanish versions, and demonstrates high reliability and validity. Both child self-report and parent proxy report come in 3 forms, based on patient age (5-7, 8-12, and 13-18 years) to account for developmental differences in responses. We used only the last 2 child and youth forms in this study. The PedsQL provides information on the physical, emotional, social, and schoolrelated aspects of HRQoL. Items generally ask how much of a problem each item has been 
during the previous month, using a 5-point response scale. Items are then reverse-scored, such that higher scores represent better HRQoL. Scores are linearly transformed to a 0-100 scale, with the 4 scale scores computed by dividing the sum of items by the number of items answered. The 3 psychosocial scores (emotional, social, school) also can be combined into a psychosocial summary score. The PedsQL has had use in several studies of populations both without apparent chronic condition and with several specific conditions (eg, cancer (21), congenital heart disease, and arthritis (22)). As examples of results on the PedsQL, using the child self-report, children with any of several chronic conditions have mean total scores of 77.19 (SD 15.53), mean physical scores of 77.36 (SD 20.36), and mean psychosocial scores of 77.10 (SD 15.84). Apparently healthy children have comparable scores of 83.00 (14.79), 84.41 (17.26), and 82.38 (15.51), respectively (20). A recent study reported mean total scores of 75.41 (SD 16.01), mean physical scores of 79.21 (SD 16.70), and mean psychosocial scores of 73.39 (SD 17.60) among 80 children with gastrointestinal disorders (10 of whom had IBD) (23). Parent report scores similarly distinguish children with and without chronic conditions.

As a measure of severity of CD, we used the Pediatric Crohn Disease Activity Index (PCDAI) (24). This measure uses a combination of history, physical examination, and laboratory values to estimate severity of CD. Scores range from 0 to 100, with higher scores reflecting more disease activity. The measure has good reliability, with validity mainly determined by comparison with a global assessment by pediatric gastroenterologists. It also is responsive to clinical change among pediatric patients with $\mathrm{CD}(25,26)$. We found no comparable measure of disease activity among pediatric patients with UC, and thus the pediatric gastroenterologists in the consortium agreed on a similar measure, based in part on a published activity index for adult UC (27). Items in the pediatric UC measure developed for this study again included stool frequency, blood in stool, abdominal cramping, temperature elevation from colitis, external manifestations (iritis, uveitis, or arthritis), and sedimentation rate, all scored similarly to the PCDAI or the adult measure. This measure has not had any previous use or validation.

In addition to these summary indices, we asked 4 general questions about the child's clinical status: their assessment of their functional status and general well-being, presence of abdominal pain or blood in the stool (at the time of assessment), and the clinician's global assessment.

\section{Analyses}

We initially carried out a confirmatory factor analysis. The confirmatory factor analysis allows us to test the hypothesis that a relationship between the observed variables and their underlying constructs exists and how well the 6-domain model describes the data. Model fit was assessed using the SAS (version 8.2, SAS Institute, Cary, NC) PROC CALIS procedure using covariance structure analysis and the maximum-likelihood estimation method. Five indices were used to determine model fit: the chi-square statistic, in which a nonsignificant chi-square close to zero supports the model (28), although because the chi-square measure is affected by sample size, a significant chi-square does not necessarily indicate poor fit (29); the adjusted goodness-of-fit index and Bentler and Bonett's (29) non-normed fit index, in which each value $>0.90$ indicates an acceptable fit; Hu and Bentler's $(28,30)$ comparative fit index, in which a value $>0.90$ (more recently, $>0.95$ ) is considered to reflect acceptable model fit; and the root mean square error of approximation, in which a value of $<0.06$ indicates an acceptable fit (28), with 0.08 being an upper bound for acceptable fit (31).

As the results will show, the initial confirmatory factor analysis was unsuccessful. We therefore conducted an exploratory factor analysis on the Impact Questionnaire responses from the children and adolescents with IBD (32). These analyses allow determination of 
latent constructs underlying the items contained in the Impact Questionnaire, not necessarily following the 6 domains proposed by the questionnaire developers. We retained factors for rotation based on the proportion of variance accounted for. Because we anticipated a relationship among the factors, we performed an oblique rotation. Simple structure was demonstrated, in that the items with high loadings on 1 factor had low loadings on the other factors. We eliminated items that did not meet a minimal loading criterion of 0.30 on any factor. We transformed each factor to a 0-100 scale to make the analyses easier to interpret. One Impact question asks whether the child missed school in the past 2 weeks. Children on vacation did not answer this question, and we imputed a value for this item from the other items loading on the same factor.

We next examined the correlations of these factors with the domains of the generic HRQoL measure (PedsQL), using Pearson product moment correlations. We then examined the associations of condition group (CD vs UC), the disease activity measures, and the other clinical variables with the factors of the IBD measure, using Student $t$ test for comparison of means for continuous variables.

\section{RESULTS}

\section{Sample}

We randomly selected 375 potential subjects from the consortium database, with the expectation of achieving a final sample of 250. Statisticians generally recommend a minimum 5:1 subject to item ratio for factor analysis (32). With 35 items on the Impact Questionnaire, we would need a minimum sample of 175 . Review by site clinicians removed 98 from this list as inactive patients without visits in the previous year. Of the remaining 277, 198 agreed to participate, 77 did not respond to mail or telephone contact, and 2 refused when approached in the clinical setting. In addition to the 198, we enrolled an additional 22 subjects in 2 sites through direct recruitment of consecutive patients meeting eligibility criteria on visits to the IBD program. Respondents and nonrespondents did not differ significantly in age, sex, or condition type (UC or $\mathrm{CD}$ ), nor did the 22 recruited differ from the other 198. Of the 220 subjects for whom HRQoL and disease severity information were collected, 73\% $(\mathrm{n}=161)$ had CD and 27\% $(\mathrm{n}=59)$ had UC (Table 1). Of the participants, $50 \%$ were female. Of the parents, $2 \%$ reported Hispanic ethnicity. Parent-reported racial data indicated a predominance of white subjects (86\%), with 10\% African American, 1\% Asian American/Pacific Islander, and 2\% of mixed, Native American, or undisclosed background. This demographic distribution reflects that of the larger cohort previously reported (18).

\section{Confirmatory and Exploratory Factor Analyses}

The initial confirmatory factor analysis was not successful. The model did not provide an acceptable fit to the data: $\chi^{2}=94.89, d f=9, P<0.0001$; adjusted goodness of fit index $=$ 0.71 ; non-normed fit index $=0.84$; comparative fit index $=0.90$; and root mean square error of approximation $=0.20$. We therefore carried out an exploratory factor analysis that, after oblique rotation and elimination of low-loading items, yielded a best fit with 4 factors for the child and adolescent data. These 4 factors accounted for $82 \%$ of the variance. We deleted 2 Impact Questionnaire items from the measure because of their low loading on all factors: "feel about test/treatments" and "how condition affects family."

Table 2 indicates the 4 factors for the child data, the loading of each item on the 4 factors, and the sources of the items from the original Impact Questionnaire. With the factor analysis, most of the Impact Questionnaire symptom items (IBD and systemic) appeared in the first factor (general well-being and symptoms). Some loaded best in the second factor, 
although not with high loading — and these items could be interpreted as reflecting feelings or emotions related to IBD symptoms. Highest-loading items for factor 2 mainly included emotional functioning items from the Impact Questionnaire. Impact Questionnaire social functioning items loaded in all 4 factors, although 3 of them appear to form a third factor in these analyses, which we have labeled "social functioning." The fourth factor, body image, aligns closely with the similar items in the Impact Questionnaire. Thus, the present analysis suggests that children and adolescents do not distinguish between systemic and IBD symptoms as categorized in the Impact Questionnaire.

Three of the factors had good internal reliability as indicated by Cronbach $\alpha$ (factor 1, 0.94; $2,0.91 ; 3,0.73)$. Factor 4 , body image, with only 3 items, had borderline acceptable reliability (0.63). Table 3 indicates the factor scores in general and for the 2 condition groups. None of the differences by condition group were statistically significant.

\section{Correlations of IBD-specific Quality of Life Factors With Generic Quality of Life Factors}

The study sample had results on the PedsQL between those for apparently healthy populations and general samples with chronic health conditions (20): mean total score, 80.89 (SD 13.71); mean physical score, 83.49 (SD 16.45); and mean psychosocial score, 79.50 (SD 13.83). We found generally high correlations between the 4 IBD factors and the factors of the PedsQL (Table 4). Of interest, the child well-being/symptom factor correlated most highly with the PedsQL health and psychosocial factors; the child emotional-functioning factor correlated most highly with the PedsQL psychosocial and emotional factors; and the child social-interactions factor correlated best with the PedsQL psychosocial and social factors.

\section{Associations of IBD-specific Quality of Life Factors With Severity and Clinical Variables}

The PCDAI data were not always collected at the same time as the QoL data, which may have arrived by mail. Of the sample, 90\% submitted PCDAI data within 2 weeks of the QoL data, but disease activity could have changed during this period. Table 5 demonstrates that children with higher PCDAI scores or higher scores on the similar UC index had lower QoL. Here, separating the PCDAI into those with scores of $0-10$ or $>10$ showed a substantial difference in mean scores on all scores except the social-interaction factor, with total scale differences of 74.0 vs $63.1(P=0.0002)$. Findings were similar for subjects with UC, despite the lack of a validated severity measure for this condition (total scale, 75.4 vs $66.9, P=$ 0.05). Table 6 indicates similar findings for the more specific clinical variables, again, with children with more symptoms or worse global assessments having worse QoL.

\section{DISCUSSION}

This study provides new information on the factor structure of a measure specifically designed to assess child views of HRQoL in diagnosed IBD. The work adds to the research on the original Impact Questionnaire by adding larger numbers of subjects and the use of factor analysis to examine characteristics of the measure. The initial confirmatory factor analysis did not support the 6 separate domains arising from the previous work mainly through interviews. Instead, our analysis indicates 4 robust factors for children and adolescents, regarding their views of their HRQoL. All of the factors except the body-image factor (with only 3 items) had good reliability. The body-image factor includes the same items as in the originally proposed domains, and has almost the same reliability in this sample as in a previous study (19); adding additional body-image items may improve the reliability. Of interest, a recent review of psychosocial functioning in pediatric IBD did not find consistent problems in body image among children with IBD (12). 
The factors' domains seem generally consistent with related domains of a well-researched measure of generic HRQoL for children and adolescents, the PedsQL. Results for the sample on the PedsQL are similar to those recently reported for a smaller $(n=42)$ population of children and adolescents with inflammatory bowel disease (33) and higher than reported for adolescents with a variety of gastrointestinal disorders (23). These results help to confirm the validity of the IBD-specific measure. Of interest, Griffiths et al compared the Impact Questionnaire with the Child Health Questionnaire (34), finding only moderate correlations between the measures (personal communication, Anne Griffiths, MD, December 2001). The differences in our study may reflect better relationships with the new factor structure, or could indicate differences between the PedsQL and the Child Health Questionnaire.

As further validation, this study indicates that the measure discriminates well among children with more and less severe or active disease. Otley et al (17) found significantly lower total Impact scores for children with more severe or active disease. Our work extends that work by showing the association most strongly with well-being and symptom scores and how these clinical variables appear to affect social and psychological functioning. The lack of a validated measure of disease activity in pediatric UC limits this clinical validation, although the consistent pattern with several severity measures supports the use of the Impact Questionnaire.

These findings suggest that children and adolescents do not distinguish among systemic symptoms, bowel-related symptoms, and treatment effects in the way that the original interviews suggested or that clinicians would. In its development over the past few years, the Impact Questionnaire has undergone changes in the allocation of specific items to domains. Other items were added because of their conceptual interest. This study provides stronger support for which items belong together and also indicates 2 items ("feel about test/ treatments" and "how condition affects family") that did not load well on any factor and likely do not add substantially to the utility of the measure in research use. The findings of this study support the use of the questionnaire, based on 4 factors and 33 items, in studies and clinical trials.

The study has several limitations. All of the subjects came from centers in the United States specializing in the care of children and adolescents with IBD and may not generally apply to all young people with IBD, although most children and adolescents with IBD receive some care in similar centers. Having 6 diverse sites likely strengthens the ability to generalize the results. Most households were middle income, and the sample lacked households that were predominantly Spanish-speaking. It also had limited racial diversity. The sample also included relatively small numbers of subjects with UC. Finally, most subjects did not demonstrate evidence of much disease activity at the time of study. In addition, the QoL assessments and disease-activity measures were not necessarily conducted at the same time. The limited numbers of subjects with active disease may have skewed the QoL findings to higher levels than would have been found in a sample with more active disease.

\section{SUMMARY}

These findings suggest that the IBD-specific measure has good psychometric properties and can be used in studies of IBD, including an increasing role in clinical trials for IBD in this age population. The analyses support categorization of IBD-related QoL into 4 domains based on 33 items. Further work should examine the stability of the IBD measure over time and its sensitivity to changes in clinical treatment, as well as its application to even larger populations (especially with larger numbers of subjects with UC) and a broader range of clinical severity. 


\section{Acknowledgments}

The authors thank Christine Eiser, PhD, Anne Griffiths, MD, and Anthony Otley, MD, for their advice.

Supported in part by National Institutes of Health grants DK 062927 and DK 060617.

\section{References}

1. Hobbs, N.; Perrin, JM.; Ireys, HT. Chronically Ill Children and Their Families. San Francisco: Jossey-Bass; 1985.

2. Baldassano RN, Piccoli DA. Inflammatory bowel disease in pediatric and adolescent patients. Gastroenterol Clin North Am. 1999; 28:445-58. [PubMed: 10372276]

3. Kim SC, Ferry GD. Inflammatory bowel diseases in pediatric and adolescent patients: clinical, therapeutic, and psychosocial considerations. Gastroenterology. 2004; 126:1550-60. [PubMed: 15168366]

4. Drossman DA, Leserman J, Mitchell CM, et al. Health status and health care use in persons with inflammatory bowel disease. A national sample. Dig Dis Sci. 1991; 36:1746-55. [PubMed: 1748045]

5. Mitchell A, Guyatt G, Singer J, et al. Quality of life in patients with inflammatory bowel disease. J Clin Gastroenterol. 1988; 10:306-10. [PubMed: 2980766]

6. Cohen RD. The quality of life in patients with Crohn's disease. Aliment Pharmacol Ther. 2002; 16:1603-9. [PubMed: 12197839]

7. Ondersma SJ, Lumley MA, Corlis ME, et al. Adolescents with inflammatory bowel disease: the roles of negative affectivity and hostility in subjective versus objective health. J Pediatr Psychol. 1997; 22:723-38. [PubMed: 9383932]

8. Wood B, Watkins JB, Boyle JT, et al. Psychological functioning in children with Crohn's disease and ulcerative colitis: implications for models of psychobiological interaction. J Am Acad Child Adolesc Psychiatry. 1987; 26:774-81. [PubMed: 3667511]

9. Gold N, Issenman R, Roberts J, et al. Well-adjusted children: an alternate view of children with inflammatory bowel disease and functional gastrointestinal complaints. Inflamm Bowel Dis. 2000; 6:1-7. [PubMed: 10701143]

10. Engstrom I, Lindquist BL. Inflammatory bowel disease in children and adolescents: a somatic and psychiatric investigation. Acta Paediatr Scand. 1991; 80:640-7. [PubMed: 1867081]

11. Engstrom I. IBD in children and adolescents: mental health and family functioning. J Pediatr Gastroenterol Nutr. 1999; 28:S28-33. [PubMed: 10204521]

12. Mackner LM, Crandall WV, Szigethy EM. Psychosocial functioning in pediatric inflammatory bowel disease. Inflamm Bowel Dis. 2006; 12:239-44. [PubMed: 16534426]

13. Ferry GD. Quality of life in inflammatory bowel disease: background and definitions. J Pediatr Gastroenterol Nutr. 1999; 28:S15-8. [PubMed: 10204518]

14. Griffiths AM, Nicholas D, Smith C, et al. Development of a quality-of-life index for pediatric inflammatory bowel disease: dealing with differences related to age and IBD type. J Pediatr Gastroenterol Nutr. 1999; 28:S46-52. [PubMed: 10204526]

15. Loonen HJ, Derkx BHF, Otley AR. Measuring health-related quality of life of pediatric patients. J Pediatr Gastoenterol Nutr. 2001; 32:523-6.

16. Loonen HJ, Derkx BHF, Last BF, et al. Development and validation of a disease specific quality of life instrument for paediatric inflammatory bowel disease: the Dutch modified questionnaire. $\mathrm{J}$ Pediatr Gastroenterol Nutr. 2001; 31:S158.

17. Otley A, Smith C, Nicholas D, et al. The IMPACT questionnaire: a valid measure of health-related quality of life in pediatric inflammatory bowel disease. J Pediatr Gastroenterol Nutr. 2002; 35:557-63. [PubMed: 12394384]

18. Heyman MB, Kirschner B, Gold B, et al. Children with early onset inflammatory bowel disease: findings of a pediatric IBD consortium registry. J Pediatr. 2005; 146:35-40. [PubMed: 15644819] 
19. Loonen HJ, Grootenhuis MA, Last BF, et al. Measuring quality of life in children with inflammatory bowel disease: The Impact-II (NL). Qual Life Res. 2002; 11:47-56. [PubMed: 12003055]

20. Varni J, Seid M, Kurtin PS. PedsQL 4.0: reliability and validity of the Pediatric Quality of Life Inventory version 4.0 generic core scales in healthy and patient populations. Med Care. 2001; 39:800-12. [PubMed: 11468499]

21. Varni JA, Burwinkle T, Katz ER, et al. The PedsQL in pediatric cancer: reliability and validity of the PedsQL and Cancer Module. Cancer. 2002; 94:2090-106. [PubMed: 11932914]

22. Varni JA, Seid M, Knight TS, et al. The PedsQL in pediatric rheumatology: validity, reliability, and responsiveness of the pediatric quality of life inventory. Arthritis Rheum. 2002; 46:714-25. [PubMed: 11920407]

23. Varni JW, Lane MM, Burwinkle TM, et al. Health-related quality of life in pediatric patients with irritable bowel syndrome: a comparative analysis. J Dev Behav Pediatr. 2006; 27:451-8. [PubMed: 17164617]

24. Hyams JS, Ferry GD, Mandel FS, et al. Development and validation of a pediatric Crohn's disease activity index. J Pediatr Gastroenterol Nutr. 1991; 12:439-47. [PubMed: 1678008]

25. Kundhal PS, Critch JN, Zachos M, et al. Pediatric Crohn Disease Activity Index: responsive to short-term change. J Pediatr Gastroenterol Nutr. 2003; 36:83-9. [PubMed: 12500001]

26. Griffiths AM, Otley AR, Hyams J, et al. A review of activity indices and end points for clinical trials in children with Crohn's disease. Inflamm Bowel Dis. 2005; 11:185-96. [PubMed: 15677913]

27. Farup PG, Hinterleitner TA, Lukas M, et al. Mesalazine $4 \mathrm{~g}$ daily given as prolonged-release granules twice daily and four times daily is at least as effective as prolonged-release tablets four times daily in patients with ulcerative colitis. Inflamm Bowel Dis. 2001; 7:237-42. [PubMed: 11515850]

28. Hu L, Bentler PM. Cutoff criteria for fit indexes in covariance structure analysis: conventional criteria versus new alternatives. Struct Equation Model. 1999; 6:1-55.

29. Bentler PB, Bonett DG. Significance tests and goodness-of-fit in the analysis of covariance structures. Psychol Bull. 1980; 88:588-606.

30. Hu L, Bentler PM. Fit indices in covariance structure modeling: sensitivity to underparameterization model misspecificiation. Psychol Methods. 1998; 3:424-53.

31. Browne, MW.; Cudeck, R. Alternative ways of assessing model fit. In: Bollen, KA.; Long, JS., editors. Testing Structural Equation Models. Newbury Park, CA: Sage; 1993. p. 136-62.

32. Gorsuch, RL. Factor Analysis. 2. Hillside, NJ: Lawrence Erlbaum; 1983.

33. Youssef NN, Langseder AL, Verga BJ, et al. Chronic childhood constipation is associated with impaired quality of life: a case-controlled study. J Pediatr Gastroenterol Nutr. 2005; 41:56-60. [PubMed: 15990631]

34. Landgraf, JM.; Abetz, L.; Ware, JE. Child Health Questionnaire: A User's Manual. 1. Boston: The Health Institute, New England Medical Center; 1996. 
TABLE 1

Characteristics of study sample

\begin{tabular}{lccc}
\hline & Ulcerative colitis N = 59 & Crohn disease N = 161 & Total N = 220 \\
\hline Age, $\mathrm{y}$ & & & \\
$8-11(\%)$ & $11(18.6)^{*}$ & $28(17.4)$ & $39(17.7)$ \\
$12-15(\%)$ & $28(47.5)$ & $66(41.0)$ & $94(42.7)$ \\
$16-18(\%)$ & $20(33.9)$ & $66(41.0)$ & $86(39.1)$ \\
Sex & & & \\
Female (\%) & $37(62.7)$ & $74(46.0)$ & $111(50.5)$ \\
Male (\%) & $22(37.3)$ & $86(53.4)$ & $108(49.1)$ \\
Race & $1(1.7)$ & & $2(0.9)$ \\
Native American (\%) & $1(1.7)$ & $1(0.6)$ & $3(1.4)$ \\
Asian American (\%) & $6(10.2)$ & $2(1.2)$ & $22(10.0)$ \\
African American (\%) & $50(84.7)$ & $16(9.9)$ & $190(86.4)$ \\
White (\%) & $1(1.7)$ & $140(87.0)$ & $2(0.9)$ \\
Other (\%) & & $1(0.6)$ & $4(1.8)$ \\
Ethnicity & $3(5.1)$ & $1(0.6)$ & \\
Hispanic (\%) & & & \\
\hline
\end{tabular}

* Percents may not total 100 because of missing data or rounding. 


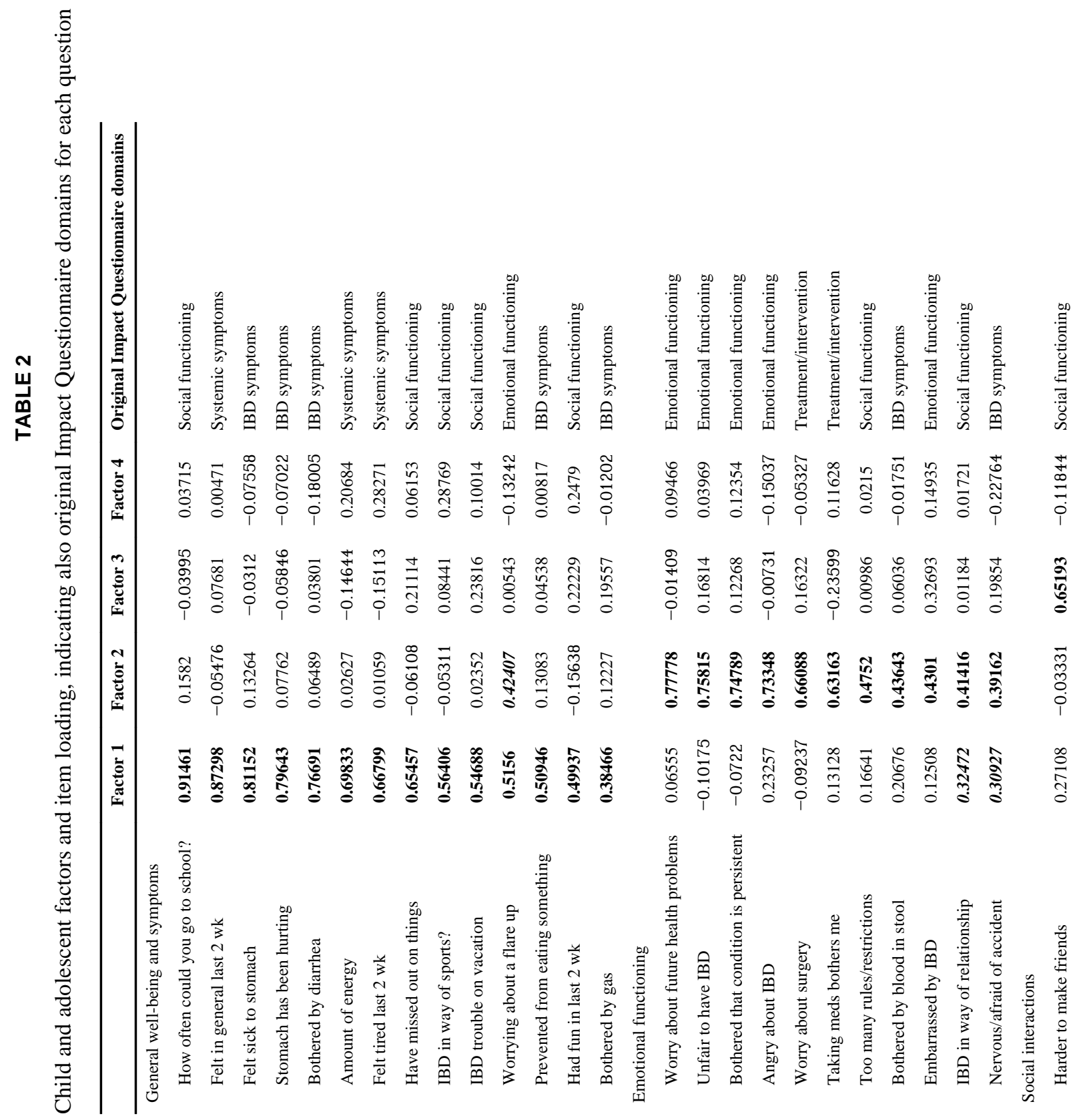




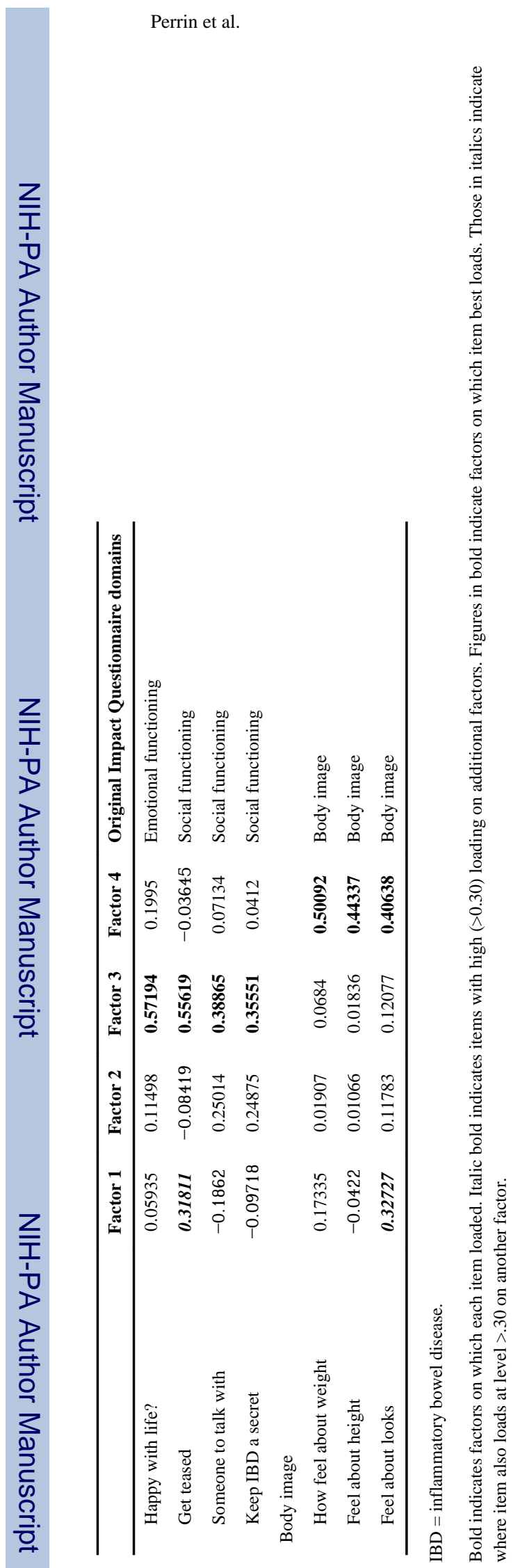

Page 12 


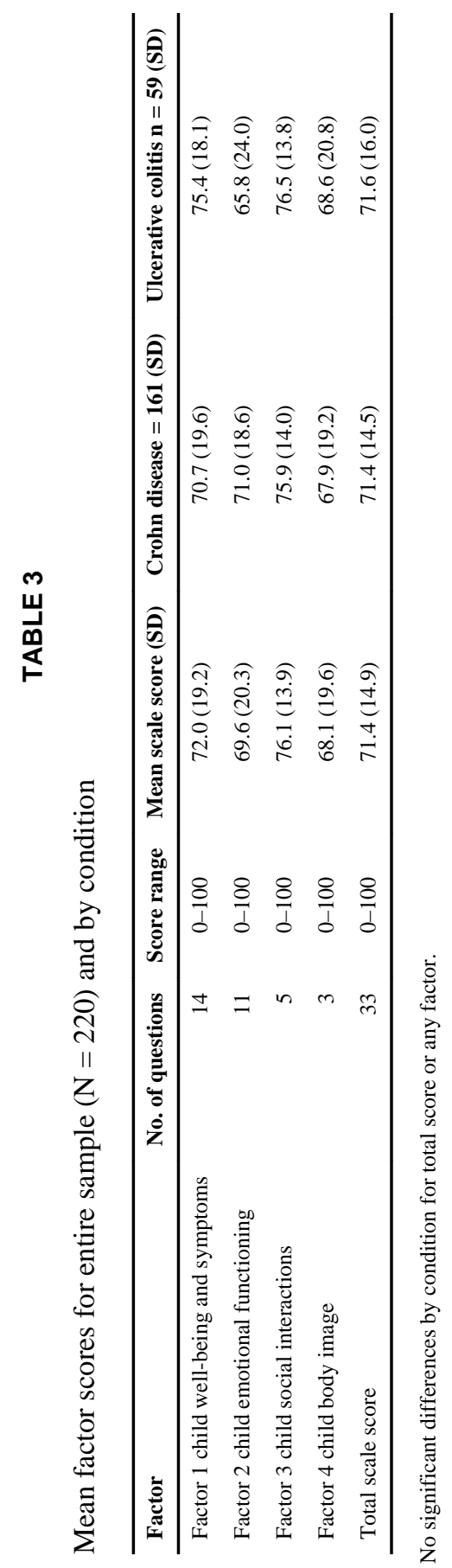

J Pediatr Gastroenterol Nutr. Author manuscript; available in PMC 2011 March 28. 
TABLE 5

Association of disease activity measures with QoL factors, for Crohn disease and ulcerative colitis

\begin{tabular}{lcccc}
\hline & PCDAI 0-10 $(\mathbf{n}=\mathbf{1 2 6})$ & PCDAI $>\mathbf{1 0}(\mathbf{n}=\mathbf{3 4})$ & $\boldsymbol{t}$ & $\boldsymbol{P}$ \\
\hline General well-being/symptoms & 74.7 & 56.0 & 5.33 & $<0.0001$ \\
Emotional functioning & 73.6 & 61.6 & 3.42 & $<0.001$ \\
Social interactions & 77.5 & 74.7 & 0.75 & $(0.45)$ \\
Body image & 70.1 & 60.1 & 2.73 & $<0.01$ \\
Total scale score & 74.0 & 63.1 & 3.86 & 0.0002 \\
\hline & UCScore $=0(\mathrm{n}=33)$ Mean Score & UCScore $>0(\mathrm{n}=24)$ Mean Score & $t$ & $P$ \\
\hline General well-being/symptoms & 80.9 & 68.6 & 2.51 & 0.02 \\
Emotional functioning & 70.1 & 60.4 & 1.52 & $(0.13)$ \\
Social interactions & 76.5 & 77.4 & -0.22 & $(0.83)$ \\
Body image & 74.0 & 61.2 & 2.36 & 0.02 \\
Total score & 75.4 & 66.9 & 2.02 & 0.05 \\
\hline
\end{tabular}

PCDAI = Pediatric Crohn Disease Activity Index UCScore = Ulcerative Colitis Score. 
TABLE 6

Associations of factors with specific clinical indicators

\begin{tabular}{|c|c|c|c|c|}
\hline & \multicolumn{4}{|c|}{ Functioning and general well-being } \\
\hline & No limitation $n=162$ & Limitations $n=38$ & $t$ & $P$ \\
\hline General well-being/symptoms & 76.8 & 53.3 & 7.71 & $<0.0001$ \\
\hline Emotional functioning & 72.5 & 56.0 & 4.63 & $<0.0001$ \\
\hline Social interactions & 77.0 & 71.5 & 2.14 & 0.03 \\
\hline Body image & 70.8 & 57.0 & 3.14 & 0.003 \\
\hline \multirow[t]{3}{*}{ Total score } & 74.3 & 59.4 & 5.78 & $<0.0001$ \\
\hline & \multicolumn{4}{|c|}{ Abdominal pain/cramping } \\
\hline & None $\mathrm{n}=141$ & Mild $/$ moderate/severe $n=60$ & $t$ & $P$ \\
\hline General well-being/symptoms & 77.4 & 60.7 & 5.46 & $<0.0001$ \\
\hline Emotional functioning & 72.2 & 63.5 & 2.77 & 0.006 \\
\hline Social interactions & 77.3 & 73.4 & 1.83 & 0.07 \\
\hline Body image & 70.5 & 62.8 & 2.29 & 0.02 \\
\hline \multirow[t]{3}{*}{ Total score } & 74.4 & 65.1 & 4.09 & $<0.0001$ \\
\hline & \multicolumn{4}{|c|}{ Clinician's global assessment } \\
\hline & Good $n=122$ & Average/poor $n=31$ & $t$ & $P$ \\
\hline General well-being/symptoms & 77.6 & 53.5 & 5.60 & $<0.0001$ \\
\hline Emotional functioning & 73.3 & 54.4 & 4.65 & $<0.0001$ \\
\hline Social interactions & 78.2 & 70.7 & 2.59 & 0.01 \\
\hline Body image & 71.5 & 59.8 & 3.06 & 0.003 \\
\hline \multirow[t]{3}{*}{ Total score } & 75.2 & 59.6 & 5.38 & $<0.0001$ \\
\hline & \multicolumn{4}{|c|}{ Blood in stool } \\
\hline & None $n=174$ & Little/a lot $n=27$ & $t$ & $P$ \\
\hline General well-being/symptoms & 74.8 & 53.6 & 4.23 & 0.0002 \\
\hline Emotional functioning & 71.1 & 56.3 & 3.55 & 0.0005 \\
\hline Social interactions & 77.5 & 65.2 & 4.40 & $<0.0001$ \\
\hline Body image & 70.0 & 58.3 & 2.27 & 0.03 \\
\hline Total score & 73.4 & 58.3 & 3.95 & 0.0004 \\
\hline
\end{tabular}

